\title{
A Multimedia Semantic Recommender System for Cultural Heritage Applications
}

\author{
Massimiliano Albanese \\ UMIACS \\ University of Maryland \\ College Park, MD 20742, USA \\ albanese@umiacs.umd.edu
}

\author{
Antonio d'Acierno \\ ISA - CNR \\ Via Roma 64, 83100 Avellino, Italy \\ dacierno.a@isa.cnr.it
}

\author{
Vincenzo Moscato, Fabio Persia \\ and Antonio Picariello \\ DIS - University of Naples \\ Via Claudio 21, 80125 Napoli, Italy \\ \{vmoscato.fabio.persia,picus\}@unina.it
}

\begin{abstract}
One of the most important challenge in the information access field is information overload. To cope with this problem, in this paper, we present a strategy for a semantic multimedia recommender system that computes customized recommendations using semantic contents and low-level features of multimedia objects, past behavior of individual users and behavior of the users' community as a whole. We have implemented a recommender prototype for browsing the Uffizi Gallery digital picture collection. Then, we investigated the effectiveness of the proposed approach, based on the users satisfaction. The obtained preliminary experimental results show that our approach is quite promising and encourages further research in this direction.
\end{abstract}

\section{INTRODUCTION}

It is well known that one of the most important challenge in the information access field is information overload. To cope with this problem, Recommender Systems help people in retrieving information that match their preferences by recommending products or services from large number of candidates and support people in making decision in various contexts: what items to buy[1], which movie to watch[2] or even who they can invite to their social networks[3]. They are especially useful in the environments with a vast amount of information where it is difficult to express the semantics of a query since they allow an automatic selection of a small subset of items that appears to fit to the user needs[4].

Formally, a recommender system deals with a set of users $U=\left\{u_{1}, u_{2}, \ldots u_{i}, \ldots u_{n}\right\}$ and a set of objects $O=\left\{o_{1}\right.$, $\left.o_{2}, \ldots o_{j}, \ldots o_{m}\right\}$, and computes, for each pair $\left(u_{i}, o_{j}\right)$, a score $r_{i, j}$ that measures the expected interest or utility of user $u_{i}$ for object $o_{j}$ using a knowledge base and a scoring (or ranking) algorithm that should also take into account that users' preferences change with context.

Each element of the user space $U$ can be defined with a profile that includes various user characteristics, such as age, gender, income, marital status, and so on; similarly, each element of the item space $O$ is defined with a set of characteristics.

For instance, in a movie recommendation application, $O$ being a collection of movies, each movie can be represented by its title, genre, director, year of release, leading actors, etc.
The utility $r$ is usually not defined on the whole $U \times O$ space, but only on some subset of it, and so the central problem is to extrapolate $r$ to the whole space $U \times O$.

In Content Based recommender systems [5], the utility $r_{i, j}$ of item $o_{j}$ is estimated using the utilities $r\left(u_{i}, o_{k}\right)$ assigned by the user $u_{i}$ to items $o_{k} \in O, k \neq j$ that are in some way similar to item $o_{j}$.

Collaborative Filtering [6] is, in the opposite, the process of filtering or evaluating items using the opinions of other people. Thus, unlike content-based recommendation methods, collaborative systems predict the utility of items $r_{i, j}$ for a particular user $u_{i}$ based on the utility $r\left(u_{h}, o_{k}\right)$ of items $o_{k}$ previously rated by other users $u_{h}$ similar to $u_{i}$. The main problem behind collaborative filtering clearly is to associate each user to a set of other users having similar profiles.

Content-based filtering and collaborative filtering may be manually combined by the end-user specifying particular features, essentially constraining recommendations to have certain content features.

More often they are automatically combined in the so called hybrid approach [7], [8] that helps to avoid certain limitations of each method (e.g., cold-start problem, overspecialization, etc...).

Recently, a new generation called semantic and social recommender systems have emerged taking advantage of the advancements in the semantic web technologies and features such as ontologies, taxonomies and social networks tagging.

Usually, semantic recommender systems are classified into three different types: vocabulary or ontology based systems, trust network based systems and context-adaptable systems that use additional ontologies depending on the manner by which it is possible to capture, represent and manage object semantics [9].

In this paper, we introduce a novel multimedia recommender system that includes a social view of the user profile and a semantic representation of the object features. The main objective of our project is to demonstrate how semantic and personalization technologies can be combined for an effective access to multimedia digital collections of museums. 
In particular, we model recommendation as a social choice problem where the set of voters and the set of alternatives both coincide with the set of objects in the data collection and describe a method that computes customized recommendations by originally combining intrinsic and semantic features of multimedia objects (low and high level similarities), past behavior of individual users and overall behavior of the entire community of users.

As motivating example, that will also be used throughout the rest of the paper, we present a typical scenario where an effective multimedia recommender system would be desirable: the case of a virtual museum that offers a web-based access to a multimedia collection of digital reproductions of Uffizi paintings in Florence.

In particular, let us consider users visiting a virtual museum and suppose that they request, at the beginning of their tour, some paintings depicting the "Holy Mary" subject.

While observing such paintings, they are attracted, for example by a Albrecht Dürer's painting entitled Madonna col Bambino (Figure 1a). It would be helpful if the system could learn the preferences of the users, based on these first interactions and predict their future needs by suggesting other paintings representing the same or related subjects, depicted by the same or other related authors or items that have been requested by users with similar preferences.
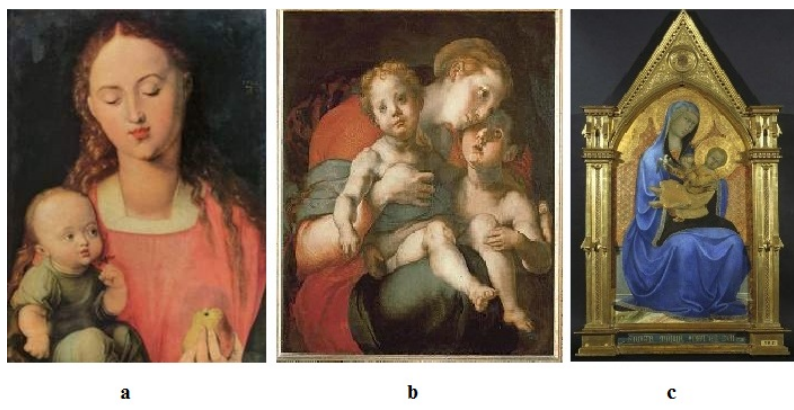

Figure 1. Paintings depicting Holy Mary

As an example, a user who is currently observing the Dürer's painting in Figure 1a might be recommended to see a Jacopo Carucci's painting entitled Madonna Bambino e San Giovannino (Figure 1b), that is quite similar to the current picture in terms of color and texture, and Madonna col Bambino by Andrea Vanni (Figure 1c), that is not similar in terms of low level features but is similar in terms of semantic content.

The paper is organized as follows. Section II describes the theoretical background and the strategy we adopted for recommendation and multimedia semantic analysis. In section III, the system architecture together with several considerations about the adopted implementation are depicted. Experimental results and conclusion are discussed in section IV and $\mathrm{V}$ respectively.

\section{A RECOMMENDATION STRATEGY BASED ON MULTIMEDIA SEMANTIC ANALYSIS}

An effective multimedia recommender system for supporting intelligent browsing of multimedia collections has the capability of reliably identify the objects that are most likely to satisfy the interests of a user at any given point of her exploration.

We have to address four fundamental questions:

1) How can we select a set of objects from the collection that are good candidates for recommendation?

2) How can we rank the set of candidates?

3) How can we capture, represent and manage semantics related to multimedia objects?

4) How can we take into account such semantics in the recommendation process?

Our recommendation algorithm is based on an importance ranking method that strongly resembles the PageRank ranking system [10] and model recommendation as a social choice problem, proposing a method that computes customized recommendations by originally combining several features of multimedia objects (low-level and semantics), past behavior of individual users and overall behavior of the entire community of users.

With respect to the theory of social choice, we assume that the set of voters and the set of alternatives coincide both with the collection. In this way we have relaxed any requirements of the Arrow's impossibility theorem and a social aggregation rule has been defined.

In addition, differently from classical citation ranking systems, where the importance of a document is influenced by the number and importance of other documents pointing to it, we do not provide explicit links between objects or any other form of citations.

Our basic idea is to assume that the fact that an object $o_{i}$ is chosen after an object $o_{j}$ in the same browsing session corresponds to $o_{j}$ voting for $o_{i}$. Similarly, the fact that an object $o_{i}$ is very similar to $o_{j}$ can also be interpreted as $o_{j}$ "recommending" $o_{i}$ (and viceversa).

Thus, our idea is to model a browsing system for $O$ as a labeled graph $(G, l)$, where $G=(O, E)$ is a directed graph and $\ell: E \rightarrow\{$ pattern, sim $\} \times R^{+}$is a function that associates each edge in $E \subseteq O \times O$ with a pair $(t, w)$, where $t$ is the type of the edge which can assume two enumerative values (pattern and similarity) and $w$ is the weight of the edge.

According to this model we can list two different cases:

- a pattern label for an edge $\left(o_{j}, o_{i}\right)$ denotes the fact that an object $o_{i}$ was accessed immediately after an object $o_{j}$ and, in this case, the weight $w_{i j}$ is the number of times $o_{i}$ was accessed immediately after $o_{j}$;

- the similarity label for an edge $\left(o_{j}, o_{i}\right)$ denotes the fact that an object $o_{i}$ is similar to $o_{j}$ and, in this case, the weight $w_{i j}$ is the similarity between $o_{j}$ and $o_{i}$. 
Thus, a link from $o_{j}$ to $o_{i}$ indicates that part of the importance of $o_{j}$ is transferred to $o_{i}$.

Given a labeled graph $(G, l)$, we can formulate the definition of recommendation grade more formally as follows.

Definition 2.1: (recommendation grade $\rho(o)$ )

$$
\forall o_{i} \in O \quad \rho\left(o_{i}\right)=\sum_{o_{j} \in P_{G}\left(o_{j}\right)} w_{i j} \cdot \rho\left(o_{j}\right)
$$

where $P_{G}=\left\{o_{j} \in O \mid\left(o_{j}, o_{i}\right) \in E\right\}$ is the set of predecessors of $o_{i}$ in $G$, and $w_{i j}$ is the normalized weight of the edge from $o_{j}$ to $o_{i}$. For each $o_{j} \in O \quad \sum_{o_{i} \in S_{G}\left(o_{j}\right)} \omega_{i j}=1$ must hold, where $S_{G}\left(o_{j}\right)=\left\{o_{i} \in O \mid\left(o_{j}, o_{i}\right) \in E\right\}$ is the set of successors of $o_{j}$ in $G$.

For instance, in a graph with 3 successors (see Figure 2) the sum $\omega_{i j}+\omega_{k j}+\omega_{m_{j}}$ must be equal to 1 .

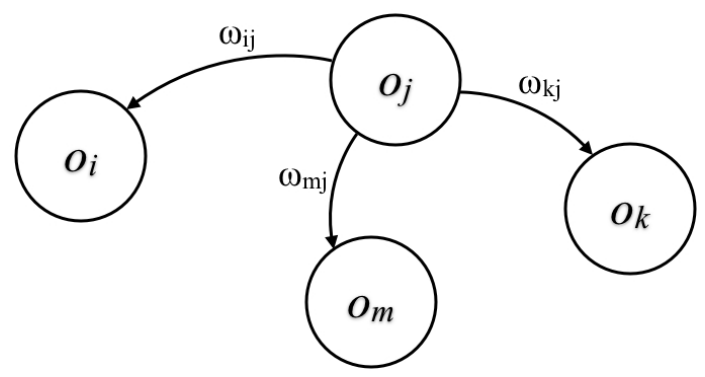

Figure 2. A graph with 3 successors

Given the iterative nature of the definition 1 , it is easy to see that the vector $R=\left[\rho\left(o_{1}\right) \ldots \rho\left(o_{n}\right)\right]^{T}$ can be computed as the solution to the following equation:

$$
R=C \cdot R
$$

where $C=\left\{\omega_{i j}\right\}$ is an ad-hoc matrix that defines how the importance of each object is transferred to other objects and can be seen as a linear combination of:

- A local browsing matrix $A_{l}=\left\{a_{i j}^{l}\right\}$ for each user $u_{l} \in U$. Its generic element $a_{i j}^{l}$ is defined as the ratio of the number of times object $o_{i}$ has been accessed by user $u_{l}$ immediately after $o_{j}$ to the number of times any object in $O$ has been accessed by $u_{l}$ immediately after $o_{j}$.

- A global browsing matrix $A=\left\{a_{i j}\right\}$. Its generic element $a_{i j}$ is defined as the ratio of the number of times object $o_{i}$ has been accessed by any user immediately after $o_{j}$ to the number of times any object in $O$ has been accessed immediately after $o_{j}$.

- A multimedia similarity matrix $B=\left\{b_{i j}\right\}$ such that:

$$
b_{i j}=\left\{\begin{array}{l}
\frac{f_{s i m}\left(o_{i}, o_{j}\right)}{\Gamma}, \text { if } f_{\text {sim }}\left(O_{i}, O_{j}\right) \geq \tau \forall i \neq j \\
0, \text { otherwise }
\end{array}\right.
$$

where $f_{\text {sim }}$ is any similarity function defined over $O$ which calculates for each couple of objects their multimedia relatedness in terms of low (features) and high level (semantics) image descriptors; $\tau$ is a threshold, and $\Gamma$ is a normalization factors which guarantees that $\sum_{i} b_{i j}=1$.

To compute $B$ matrix, we have decided to adopt 4 sets of multimedia features (Tamura descriptors, MPEG7 color-based descriptors, MPEG-7 edge-based descriptors, MPEG-7 color layout- based descriptors and all MPEG7 descriptors [11]) and the related similarity metrics have been implemented by LIRE tool.

In addition, we exploit specific image metadata (artist, genre and subject) and the semantic similarity has been computed used the most diffused metrics for semantic relatedness of concepts based on a vocabulary (Li-BandarMcLean, Wu-Palmer, Rada, Leacock-Chodorow [12]).

In particular the semantic similarity combines similarities among artists, genres and subjects obtained by using a fixed taxonomy which part is shown in fig. 3 .

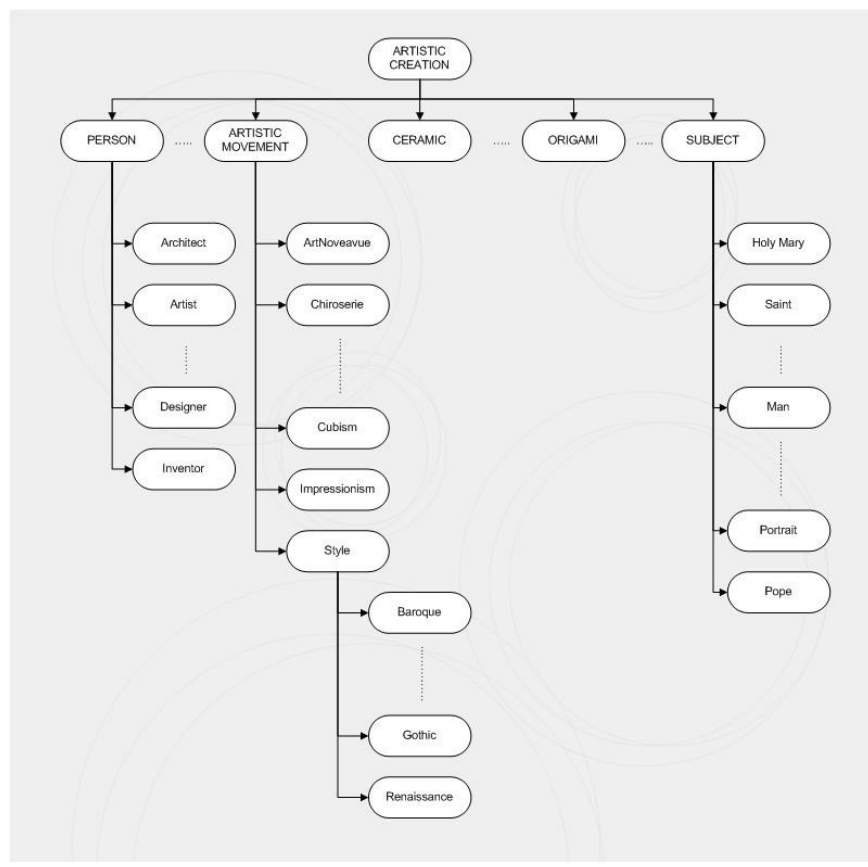

Figure 3. The used taxonomy

To obtain the combination of low and high level descriptors that maximize recall and precision in the retrieval process, we have adopted the following experimental protocol.

1) We performed on the system a set of 50 hybrid queries, each one specified by a target image (not present in the collection) and only by one (that user considers more important) of the related metadata (genre, artist and subject).

2) For each query, the system returned the top key of the 50 images that are most similar to the target one and an image in the result set is considered relevant if at 
least two of the related metadata are the same with respect to those associated to the query object.

3) We computed on the base of relevant objects the recall, precision and $F 1$ measures first by using separately each one of the 7 similarity metrics and then by considering two kinds of combinations of low and high level similarities (weighted sum and Sugeno fuzzy integral) with the related weights opportunely chosen in the tuning phase.

Figure 4 shows the obtained results and how: (i) high level similarities better capture the query semantics (see fig. 4.a), (ii) the best combination between high and low level descriptors is Sugeno fuzzy integral of Li and MPEG-7 color layout- based similarities in order to have more high values of precision, and Sugeno fuzzy integral of Wu-Palmer and MPEG-7 color based similarities in order to have more high level values of recall (see fig. 4.b, we use this combination for matrix $B$ computation).

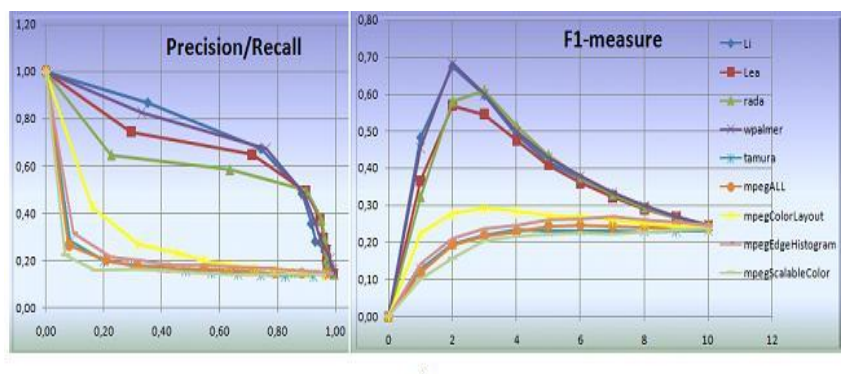

a)

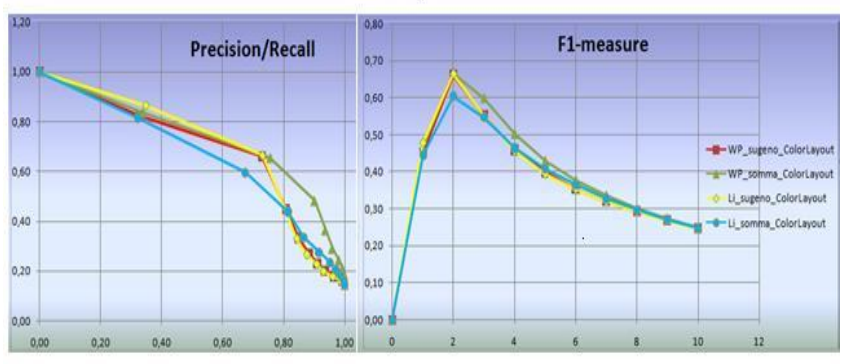

b)

Figure 4. Setting of multimedia descriptors that better take into account image semantics

So far we have a suitable manner to represent object features and to compare the related similarity also considering semantics in terms of object metadata; now, our main goal is to compute customized rankings for each individual user. We can then rewrite equation 2 as follows:

$$
R_{l}=C \cdot R_{l}
$$

where $R_{l}=\left[\rho\left(o_{1}\right) \ldots \rho\left(o_{n}\right)\right]^{T}$ is the vector of recommendation grades, customized for a user $u_{l}$.

We note that solving equation 4 corresponds to find the stationary vector of $C$, i.e., the eigenvector with eigenvalue
1. We demonstrated in [13] that $C$, under certain assumptions and transformations, is a real square matrix having positive elements, with a unique largest real eigenvalue and the corresponding eigenvector has strictly positive components. In such conditions, equation 4 can be solved used the Power Method algorithm.

Assuming that a user $u_{l}$ is currently watching object $o_{j}$, we can define the final set of candidate recommendations as follows:

$$
C_{j}=\bigcup_{k=1}^{M}\left\{o_{i} \in O \mid A_{i j}^{k}>0\right\} \cup\left\{o_{i} \in O \mid B_{i j}>0\right\}
$$

The set of candidates includes the objects that have been accessed by at least one user within $k$ steps from $o_{j}$, with $k$ between 1 and $M$, and the objects that are most similar to $o_{j}$.

In figure 5 there is an easy example of how to compute the set of candidates in the case the collection has only ten paintings and the most similar images to the current image are only four. As we can see, after selecting the most similar images, from each of these the images accessed within 2 steps are selected.

\section{THE SYSTEM}

In this section, we describe a case study in the cultural heritage domain for a web recommendation system that provides browsing facilities for multimedia collection of the Uffizi Gallery paintings. In particular, our recommender helps the users for finding paintings of interest from a large set of choices, proposing a set of suggestions for each observed object; the recommendations are computed combining the of user's behaviour with low and high level image descriptors, following the widely described approach.

We use a memory-based algorithm so that low and high level similarities are evaluated once; this reflect the unchanging nature of these measures while, clearly, if we add new paintings, similarity matrices have to be conveniently updated. Instead, to capture the dynamic nature of user's behaviour, we periodically recompute connection matrices; specifically, each connection matrix is updated as soon the browsing session ends.

To solve the cold start problem, when there is no information about user's behaviour, our system uses low or/and high level similarities, in addition to the extracted behaviour of the whole community. For new items, of course, recommendation is based just on similarities.

Our data collection consists of 474 digital reproductions of paintings, which in turn belong to 144 artists (Botticelli, Giotto, and so on), grouped into 16 artistic genres from Gothic to Contemporary art.

Each painting is also linked to a pair of subjects, which have been chosen among the 47 available ones; such an information roughly represents what the painting represents. 

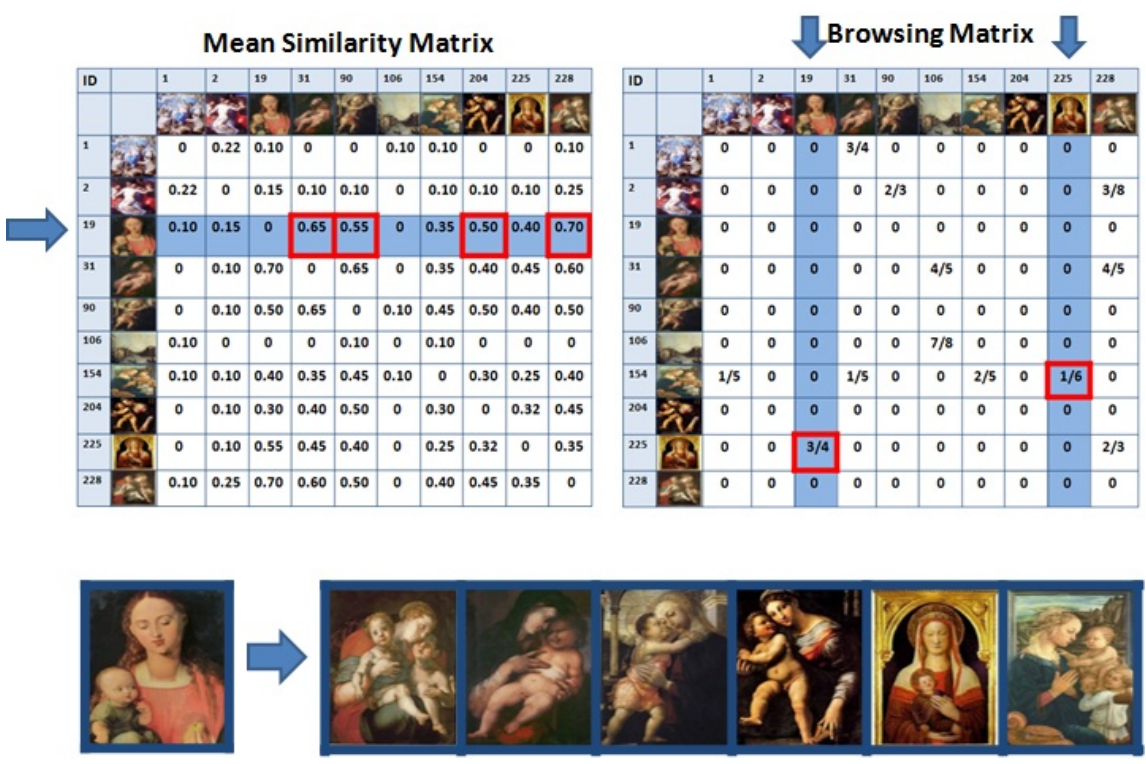

Figure 5. How to compute the set of candidates

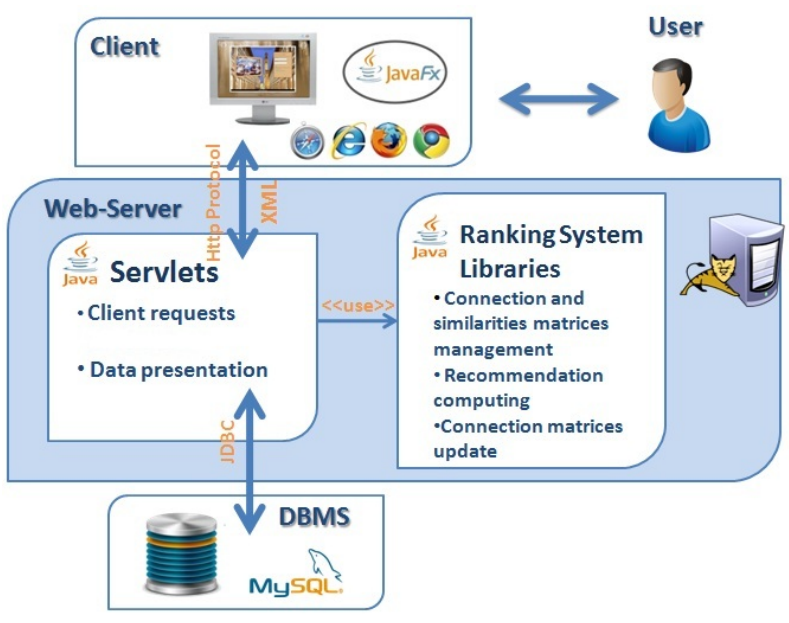

Figure 6. Uffizi Gallery: Architecture

A user interacts with our system (figure 6) using a web browser that communicates with the server by means of straight http requests.

The presentation logic is based on JavaFX technology that allows to interact with users using advanced graphical functionalities, while client requests are elaborated by JAVA Servlets and results are sent to the client in form of $X M L$ data (according to the Service Oriented Architecture paradigm). The core functionality of the system, the recommendation process, can be described as follow.

As soon as a user interacts with the system, the core process starts defining the set $\bar{S}$ of candidates for the recommendation by considering the union of:
1) the set of paintings which are the most similar to the current one, according the similarity matrices;

2) the set of paintings which have been accessed by at least one user within a certain number of steps from the current one; to reach this goal, if the user is logged in and there exits the related user connection matrix, the past user's behaviour is considered; otherwise the global connection matrix is taken into account.

$\bar{S}$, of course, takes into account the user's context and, thus, the $C$ matrix is built just referencing the elements belonging to such set; the Power Method is then used to compute the ranking vector, that is in eventually exploited to recommend new paintings.

At the end of each browsing session, the system updates the connection matrix extracting a set of pairs (image to be accessed - accessed image); for instance, if once observed the $j$-th painting the user sees the $i$-th painting, then an occurrence will be added to the $A_{i j}$ element in the corresponding connection matrix.

From the final users perspective, the client application has the following features:

- a set of forms to provide users log in or registration;

- a gallery to visualize images which are returned after a search by author, subject or artistic genre;

- visualization of an image and of the related information and presentation of recommended images;

- storing of user session with the information related to the browsing patterns.

In figure 7 we report a screenshot of the client application. 


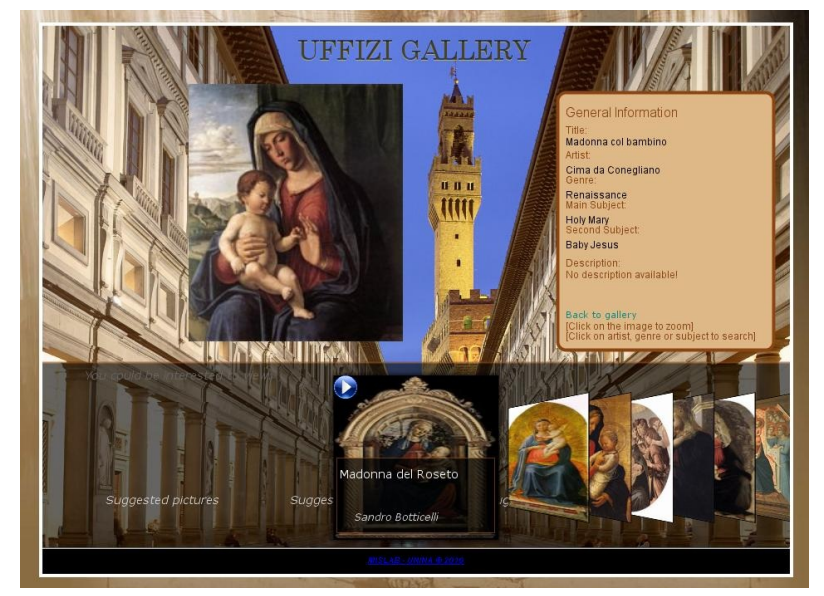

Figure 7. Uffizi Gallery: client-side application

\section{Preliminary Experimental Results}

Recommender systems are complex applications that are based on a combination of several models, algorithms and heuristics. This complexity makes evaluation efforts very difficult and results are hardly generalizable, which is apparent in the literature about recommender evaluation. Previous research work on recommender system evaluation has mainly focused on algorithm accuracy, especially objective prediction accuracy. More recently, researchers began examining issues related to users subjective opinions and developing additional criteria to evaluate recommender systems.

Starting from these considerations and according to the literature trend, we decided to give more importance to a user-centric evaluation and the proposed evaluation strategy aims at measuring the effectiveness of the system in terms of the user satisfaction with respect to assigned browsing tasks.

In particular, we evaluated the impact of our system on the users and compared its performances with respect to another existing system for organizing and browsing large photo collection (Picasa Web Albums where taxonomies were implemented as albums, folders and descriptions.), which does not take into account browsing behavior of users and intrinsic features of the multimedia objects. Our goal was to establish how helpful our system was to provide an exploration of digital reproductions of paintings. Moreover from these experiments we wanted to understand how helpful recommendations offered by our recommender system were to address users toward paintings which satisfied their interests.

In the first stage of our experiments we asked a group of 20 people to browse the digital collection of paintings, with the assistance of our recommender system, and complete several browsing tasks of different complexity.

This group consisted of 10 not-expert users on art, 5 medium expert users on art and 5 expert users on art. After this test, we asked them to browse once again the same collection of paintings using Picasa.

We defined four browsing tasks, as described below:

1) Low Complexity tasks $\left(T_{1}\right)$ : explore at least 10 paintings of Renaissance style.

2) Medium Complexity tasks $\left(T_{2}\right)$ : explore at least 20 paintings of Renaissance style that have Holy Mary as their subject.

3) High Complexity tasks $\left(T_{3}\right)$ : explore at least 20 paintings of Renaissance style with subject Holy Mary and with a predominance of dark blue color.

4) Very High Complexity tasks $\left(T_{4}\right)$ : explore at least 3 paintings of Renaissance style with subject Holy Mary and with a predominance of dark blue color whose author is Botticelli.

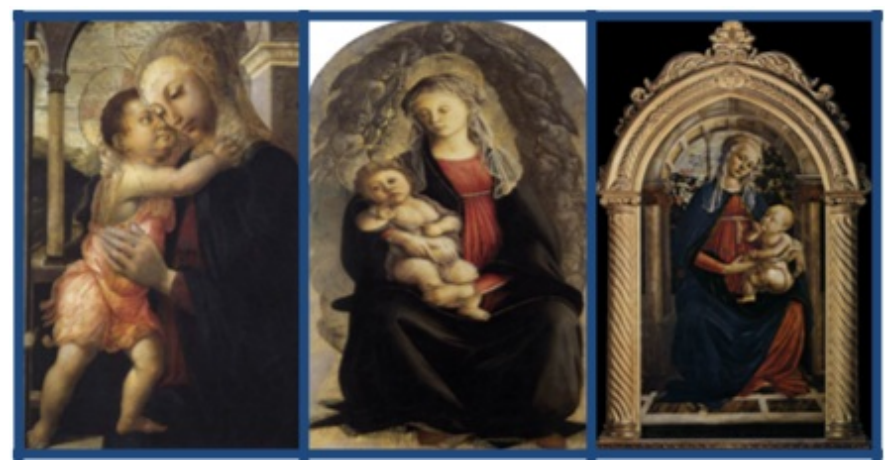

Figure 8. A possible result of $T_{4}$ task of browsing.

For instance a possible result of the tasks $T_{4}$ is showed in fig.8, where from left to right we have "Madonna della Loggia", "Madonna in gloria di Serafini" and "Madonna del Roseto", which are 3 paintings of Renaissance style with subject Holy Mary and with a predominance of dark blue color, whose author is Botticelli.

Two strategies were used to evaluate the results of this experiment: (i) empirical measurements of access complexity in terms of mouse clicks and time; (ii) TLX (NASA Task Load Index factor).

With respect to the first strategy, we measured the following parameters:

- access Time $\left(\boldsymbol{t}_{\boldsymbol{a}}\right)$ : the average time spent by the users to request and access all the objects for a given class of tasks;

- number of clicks $\left(\boldsymbol{n}_{\boldsymbol{c}}\right)$ : the average number of clicks necessary to collect all the requested objects for a given class of tasks.

Table I reports the average values of $t_{a}$ and $n_{c}$ for both Picasa and our system (Uffizi Gallery), for each of the four task complexity levels defined.

We then asked the users to express their opinion about the capability of Picasa and our system respectively to provide 


\begin{tabular}{|l|c|c|r|}
\hline Task Class & System & $\boldsymbol{T}_{\boldsymbol{a}}(\mathrm{sec})$ & $\boldsymbol{N}_{\boldsymbol{c}}$ \\
\hline Low Complexity & Uffizi Gallery & 142 & 24 \\
\hline Low Complexity & Picasa & 120 & 19 \\
\hline Medium Complexity & Uffizi Gallery & 216 & 59 \\
\hline Medium Complexity & Picasa & 315 & 66 \\
\hline High Complexity & Uffizi Gallery & 369 & 97 \\
\hline High Complexity & Picasa & 446 & 102 \\
\hline Very High Complexity & Uffizi Gallery & 552 & 126 \\
\hline Very High Complexity & Picasa & 674 & 159 \\
\hline
\end{tabular}

Table I

COMPARISON BETWEEN OUR SYSTEM AND PICASA IN TERMS OF $t_{a}$ AND $n_{c}$ AVERAGE VALUES

an effective user experience in completing the assigned browsing tasks by the TLX evaluation. Specifically, TLX is a multi-dimensional rating procedure that provides an overall workload score based on a weighted average of ratings on six sub-scales: mental demand, physical demand, temporal demand, own performance, effort and frustration. The lower TLX scores, the better they are.

We obtained the average results scores for each of three categories of users reported in table .IV.

As we can note by our results, according to not-expert users on art, our system outperforms Picasa in every subscale, because they consider very helpful the suggestions provided in order to orientate them in the exploration of our images dataset. Instead, in expert users opinion and according to medium expert users on art, our system outperforms Picasa in every sub-scale except for mental demand and performance, this happens because an expert user on art considers not always useful automatic suggestions to complete the assigned browsing tasks, thus sometimes he considers faster and easier to use Picasa rather than our system (Picasa offers a more complete vision on the whole collection of images and an easier way to browse all the paintings).

However, overall, our system allows to provide a better (less frustrating) user experience during her browsing tasks.

\section{Conclusions}

In this paper we proposed a multimedia semanti approach to recommendation in browsing systems, based on a method that computes customized recommendations by combing in an original way intrinsic features (semantic contents and low-level features) of the objects, past behavior of individual users and behavior of the users' community as a whole. In particular, we realized a recommender system which helps users to browse digital reproductions of Uffizi paintings, providing them suggestions computed by our novel method for recommendations. Then we investigated the effectiveness of the proposed approach in the considered scenario, based on the users satisfaction.

Experimental results showed that our approach is promising and encourages further research in this direction.

\section{ACKNOWLEDGMENTS}

This work has been carried out partially under the financial support of the Programma FARO (Finanziamento per l'Avvio di Ricerche Originali) in the framework of the LATINO Project: Un sistema innovativo di LocAlizzazione e Tracciamento di INdividui per l'Ottimizzazione di percorsi in ambienti indoor.

\section{REFERENCES}

[1] X. Zhang and H. Wang, "Study on recommender systems for business-to-business electronic commerce," Communications of the IIMA, vol. 5, pp. 53-61, 2005.

[2] S. Qin, R. Menezes, and M. Silaghi, "A recommender system for youtube based on its network of reviewers," in SocialCom/PASSAT, A. K. Elmagarmid and D. Agrawal, Eds. IEEE Computer Society, 2010, pp. 323-328.

[3] P. Kazienko and K. Musial, "Recommendation framework for online social networks," in Advances in Web Intelligence and Data Mining, ser. Studies in Computational Intelligence, M. Last, P. S. Szczepaniak, Z. Volkovich, and A. Kandel, Eds. Springer, 2006, vol. 23, pp. 111-120.

[4] S. Perugini, M. A. Gonçalves, and E. A. Fox, "Recommender systems research: A connection-centric survey," J. Intell. Inf. Syst., vol. 23, pp. 107-143, September 2004. [Online]. Available: http://dx.doi.org/10.1023/B:JIIS.0000039532.05533.99

[5] M. Pazzani and D. Billsus, "Content-Based Recommendation Systems," 2007, pp. 325-341. [Online]. Available: http://dx.doi.org/10.1007/978-3-540-72079-9_10

[6] G. Adomavicius and A. Tuzhilin, "Toward the next generation of recommender systems: A survey of the state-of-the-art and possible extensions," IEEE Transactions on Knowledge and Data Engineering, vol. 17, pp. 734-749, 2005.

[7] L. Si and R. Jin, "Unified filtering by combining collaborative filtering and content-based filtering via mixture model and exponential model," in Proceedings of the thirteenth ACM international conference on Information and knowledge management, ser. CIKM '04. New York, NY, USA: ACM, 2004, pp. 156-157. [Online]. Available: http://doi.acm.org/10.1145/1031171.1031201

[8] P. Kazienko and P. Kolodziejski, "Personalized integration of recommendation methods for e-commerce," Journal of Computer Science and Applications, vol. 3, 2006.

[9] E. Peis, J. del Castillo, and J. Delgado-Lopez, "Semantic recommender systems: analysis of the state of the topic," in Proc. of Hipertext 2008, 2008.

[10] L. Page, S. Brin, R. Motwani, and T. Winograd, "The pagerank citation ranking: Bringing order to the web." Stanford InfoLab, Technical Report 1999-66, November 1999, previous number = SIDL-WP-1999-0120. [Online]. Available: http://ilpubs.stanford.edu:8090/422/

[11] M. Lux and A. Chatzichristofis, "Lire: Lucene image retrival - an extensible java cbir library," in Proceedings of the 16th ACM International Conference on Multimedia, 2008, pp. 1085-1088. 


\begin{tabular}{|c|c|c|c|c|c|c|}
\hline & \multicolumn{2}{|c|}{ Experts } & \multicolumn{2}{c|}{ Medium Experts } & \multicolumn{2}{c|}{ Not Experts } \\
\hline TLX factor & Our System & Picasa & Our System & Picasa & Our System & Picasa \\
\hline Mental demand & 20.5 & 18.5 & 28.2 & 27 & 30 & 38 \\
\hline Physical demand & 23 & 28 & 25.3 & 30 & 26.2 & 40 \\
\hline Temporal demand & 26.2 & 31.1 & 27.6 & 32 & 27 & 31 \\
\hline Effort & 23.3 & 28.2 & 26.7 & 37.2 & 32.3 & 46.4 \\
\hline Perfomances & 70.1 & 66.3 & 70.6 & 69.3 & 60.2 & 70.1 \\
\hline Frustation & 20.5 & 29 & 22.3 & 30.5 & 23.4 & 31.6 \\
\hline
\end{tabular}

Table II

COMPARISON BETWEEN OUR SYSTEM AND PICASA IN TERMS OF TLX FACTORS FOR EACH CATEGORY OF USERS

[12] A. Budanitsky and G. Hirst, "Semantic distance in wordnet: An experimental, application oriented evaluation of five measures," in Proceedings of the Workshop on WordNet and other Lexical Resources, 2001.

[13] M. Albanese, A. d'Acierno, V. Moscato, F. Persia, and A. Picariello, "Modeling recommendation as a social choice problem," in Proceedings of the fourth ACM conference on Recommender systems, ser. RecSys '10. New York, NY, USA: ACM, 2010, pp. 329-332. [Online]. Available: http://doi.acm.org/10.1145/1864708.1864779 